\title{
Effectiveness of early cardiology undergraduate learning using simulation on retention, application of learning and level of confidence during clinical clerkships
}

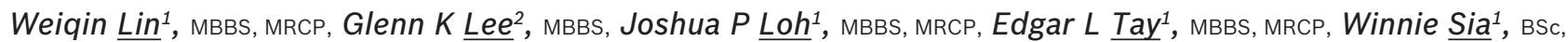
Tang-Ching $\underline{L a u}^{2,3}, \mathrm{MBBS}$, PhD, Shing-Chuan $\underline{\mathrm{Hoo}}^{3}$, MBBS, PhD, Kian-Keong $\underline{\text { Poh }}{ }^{1,3}$, FRCP, FACC

\begin{abstract}
INTRODUCTION This study aimed to assess the effectiveness of the use of a cardiopulmonary patient simulator in the teaching of second-year medical students. Effectiveness was measured in terms of the extent of knowledge retention and students' ability to apply the skills learned in subsequent real-life patient contact.

METHODS In this study, ten third-year medical students who had previously undergone simulator training as part of their second-year curriculum underwent an objective structured clinical examination (OSCE) and a multiple-choice question (MCQ) test to assess their ability to apply the knowledge gained during the simulator training when dealing with real patients. The performance of this group of students was compared with that of a group of ten fourth-year medical students who did not undergo simulation training.

RESULTS Although the third-year medical students performed well in the OSCE, they were outperformed by the group of fourth-year medical students, who had an extra year of clinical exposure. The MCQ scores of the two groups of students were similar. Post-simulation training survey revealed that students were generally in favour of incorporating cardiopulmonary simulator training in the preclinical curriculum.

CONCLUSION Cardiopulmonary simulator training is a useful tool for the education of preclinical medical students. It aids the translation of preclinical knowledge into real-life clinical skills.
\end{abstract}

Keywords: cardiology, medical education, medical student, physical examination, simulation training

\section{INTRODUCTION}

The use of simulation in education has been increasingly prevalent in recent years and medical education is no exception to this phenomenon. While experiential-based learning is highly valued in medical education, ${ }^{(1,2)}$ it is limited by patient safety considerations $^{(3-7)}$ and the relatively low access medical students have to patients. The use of simulation training in clinical education may circumvent such constraints. In fact, high-fidelity medical simulations have been shown to be educationally effective, and simulation-based education has been shown to complement traditional bedside teaching. ${ }^{(8)}$ Most studies report that medical students who underwent simulation training had improved test scores in assessments that measure clinical skill and knowledge. ${ }^{(9-14)}$ Furthermore, simulation training may be more practical in certain situations; for example, during an epidemic, students may not be allowed to practise physical examination of patients in wards. One study performed during the severe acute respiratory syndrome epidemic in 2003 explored the utility of teaching cardiac auscultation without patient contact. ${ }^{(15)}$ For that study, patients with echocardiographically proven heart disease had their heart sounds recorded on electronic stethoscopes. After these recorded sounds were validated, they were used to teach students during simulated bedside sessions. The objective outcome measures and subjective feedback in that study demonstrated that the method was effective and well-received by the students. ${ }^{(15)}$ However, there is still a lack of conclusive research on the cost-benefit factors of the use of simulation in education, and a lack of evidence on the real impact simulation has on actual patient care. ${ }^{(16)}$

The simulator employed in the teaching of the cardiovascular system is Harvey ${ }^{\circledR}$ The Cardiopulmonary Patient Simulator (Laerdal Medical, Stavanger, Norway). The simulator is a full-size manikin that is able to realistically simulate more than 30 cardiac conditions (through various clinical signs such as heart sounds, murmurs, jugular venous pulsation, nature of apex beat and breath sounds) at the touch of a button. It can be structured to teach students not only common and less complex conditions, but also rare and complex diseases. The main goal of the learning activity using the simulator is for students to understand the functionality of the cardiovascular system under normal physiological conditions and to appreciate how it is affected in various disease processes. The Harvey simulator has been shown to be effective in enabling students to achieve a reasonable level of competency in the skills taught. ${ }^{(17)}$ As such, it has been accredited and recommended for medical education by many institutions worldwide. In fact, the British Heart Foundation purchased a Harvey simulator for each medical school in the United Kingdom. ${ }^{(18)}$

The undergraduate curriculum of the Yong Loo Lin School of Medicine, National University of Singapore, Singapore, was revised in 2008 to integrate the content covered and increase the

${ }^{1}$ Department of Cardiology, National University Heart Centre, ${ }^{2}$ Department of Medicine, National University Health System, ${ }^{3}$ Yong Loo Lin School of Medicine, National University of Singapore, Singapore

Correspondence: A/Prof Poh Kian-Keong, Associate Professor, Department of Cardiology, National University Heart Centre, 1E Kent Ridge Road, NUHS Tower Block, Level 9 , Singapore 119228. kian_keong_poh@nuhs.edu.sg 
relevance of the basic science curriculum, so as to better equip students for the clinical phases of medical school. A key feature of the revised curriculum is the incorporation of simulation in the systems-based preclinical modules. The cardiovascular system module is one such example. The use of the Harvey simulator was incorporated into the teaching-learning schedule of each group of medical students, so as to expose them to core mustknow conditions in cardiovascular diseases through hands-on practice sessions. The Harvey simulator allows students to practise examination of a 'patient' with varied signs, some of which may be rare in clinical practice. In other words, it may take many weeks or months for students to encounter all the same signs (i.e. to gain the same experience via simulation training) through the examination of real patients in normal clinical practice.

The present study was designed to evaluate the effectiveness of the revised curriculum with Harvey in enhancing medical students' knowledge retention and their ability to apply the knowledge and skills acquired (during the simulation training) to actual clinical cases. The study also included a survey of the students' perception of their level of confidence in applying what was learned to their future clinical clerkships.

\section{METHODS}

A total of ten students in their third year of medical study were recruited to undergo an objective structured clinical examination (OSCE) and a multiple-choice question (MCQ) test. These students had undergone the revised curriculum that included Harvey simulation and completed two years of basic science training, but have yet to undergo internal medicine or paediatric rotations. The results of the OSCE and MCQ test were examined, and a post-examination survey (see Appendix) was conducted to examine whether the simulator training was able to adequately prepare the students for subsequent real patient encounters and clinical examinations.

All of the ten students had attended two 3-hour, hands-on simulation training sessions when they were second-year medical students. In the first session (an introductory session), the students familiarised themselves with the simulator. Facilitators guided the students through general scenarios and discussions about the physiological and anatomical bases of relevant clinical signs. Students were given the opportunity to go through the clinical signs repeatedly until they were familiar with them. The second session was a revision of the murmurs and clinical scenarios. After these two short sessions with the simulator, the students were not exposed to cardiology or internal medicine rotations for about a year, up to the time they participated in the present study. Another ten students who were in their fourth year of medical study were recruited to undergo the same OSCE and MCQ test without a post-test survey.

Both groups of students were required to undergo five OSCE stations that examined core clinical competencies in cardiology. At each OSCE station, students were required to examine a real patient with one of the following cardiac conditions: aortic stenosis, mitral stenosis, mitral regurgitation, mitral valve prolapse and a prosthetic valve. The students presented their findings to an examiner, who then graded them based on an objective marking
Table I. Comparison of performances between the third- and fourth-year medical students in the objective structured clinical examination (OSCE) stations and multiple-choice question (MCQ) test.

\begin{tabular}{lccc}
\hline Variable & \multicolumn{2}{c}{ Mean score (\%) } & p-value \\
\cline { 2 - 3 } & $\begin{array}{c}\text { Third-year } \\
\text { medical } \\
\text { students } \\
(\mathbf{n}=\mathbf{1 0})\end{array}$ & $\begin{array}{c}\text { Fourth-year } \\
\text { medical } \\
\text { students } \\
(\mathbf{n}=\mathbf{1 0})\end{array}$ & \\
\hline $\begin{array}{l}\text { OSCE - examination } \\
\text { technique score }\end{array}$ & $65 \pm 14$ & $79 \pm 8$ & 0.020 \\
$\begin{array}{l}\text { Overall OSCE score } \\
\text { MCQ test score }\end{array}$ & $54 \pm 9$ & $70 \pm 7$ & 0.006 \\
Overall score* & $50 \pm 15$ & $56 \pm 13$ & 0.200 \\
\hline
\end{tabular}

Note: In the present study, the third-year medical students had undergone simulation training sessions with Harvey, while the fourth-year medical students had not. *Includes both OSCE and MCQ test results.

scheme comprising the following components: (a) student's general approach and rapport with the patient; (b) student's examination technique; and (c) student's ability to pick up on clinical signs and carry out a short discussion of the case. This is similar to the validated assessment template that the Yong Loo Lin School of Medicine uses for its professional examinations. The OSCE examiners were practicing cardiologists who had examined and verified the clinical findings in the patient. Each student was tested on the same patient and graded by the same examiner, which translates into higher consistency and greater objectivity in the assessment. Upon completion of the OSCE, the third-year students were asked to complete a survey on the utility and limitations of the Harvey simulation sessions.

The MCQ test comprised 32 questions that primarily examined the students' understanding of important physical signs, including pathophysiology in cardiology and its relation to various conditions. The questions were in the 'best answer out of four choices' format and the students were allocated an hour to complete the test. There was no negative marking.

\section{RESULTS}

The OSCE stations were scored according to the following four categories: (a) general approach; (b) examination technique; (c) signs; and (d) synthesis. The third-year medical students, who were examining real patients for the first time, did reasonably well in the OSCE. The OSCE and MCQ test results of the third- and fourthyear medical students are shown in Table I. In the examination technique category, the mean score of the third-year medical students was $65 \% \pm 14 \%$, while that of the fourth-year medical students was $79 \% \pm 8 \%$. The fourth-year medical students had undergone one extra year of medical training, including a rotation in internal medicine, prior to undergoing the OSCE. In terms of the combined scores of all the OSCE components, the mean scores for the third- and fourth-year medical students were $54 \% \pm 9 \%$ and $70 \% \pm 7 \%$, respectively.

For the MCQ test, the mean scores of the third- and fourth-year medical students were $50 \% \pm 15 \%$ and $56 \% \pm 13 \%$, respectively. However, this difference was not found to be statistically significant $(p=0.20)$. Combining the OSCE results with the MCQ 
test results, the overall mean scores for the third- and fourth-year medical students were $52 \% \pm 8 \%$ and $65 \% \pm 8 \%$, respectively.

Based on the post-examination survey conducted on the third-year medical students who participated in the simulation sessions, the students generally felt that the sessions provided them with greater opportunity for practice and boosted their confidence for clinical rotations. Although there were drawbacks to the simulation sessions such as its limited duration, the large class size and the lack of variability in the severity of clinical signs (which is normally seen in real patients), all the students had positive comments on it.

\section{DISCUSSION}

The present study looked at whether a simulation module would help medical students in their clinical rotations and subsequent learning in the clinical years. Bearing in mind that in this study, the OSCE represented the third-year medical students' first contact with real patients who had cardiovascular signs, the students did reasonably well with regard to their examination technique. This shows that training with the Harvey simulator in the preclinical years helps students acquire foundational skills in cardiology and medicine, and that this form of learning is a useful adjunct in medical education.

Comparing the OSCE results of the third-year medical students with that of the fourth-year medical students, we found that most of the third-year medical students managed to score well in the examination technique category, which is the category that Harvey simulation specifically trains for. The third-year medical students scored reasonably well despite not having gone through any formal clinical rotation in internal medicine. They relied mainly on the skills that they had picked up during the simulation training. Compared to the third-year medical students, the fourth-year medical students scored better in the skill sets tested during the OSCE and achieved better overall OSCE scores. This is not unexpected in light of the extra year of clinical exposure that the fourth-year medical students had gone through. Although the fourth-year medical students had varied degrees of exposure to cardiac cases during their clinical postings in various hospitals, the types of patients that were tested in the OSCE were core clinical cases, as indicated in the undergraduate curriculum. During the course of their posting, the fourth-year medical students would have had sufficient opportunities to see patients with the conditions tested in the OSCE, thus enabling them to do well in the OSCE stations.

It was interesting to note that although the present study examined what third-year medical students had learned in their second year, they appeared to be able to retain what they had learned even after a year and were able to perform reasonably well in the OSCE. This is despite the students having undergone only two sessions of simulation teaching for the cardiovascular system.

In the present study, the scores for the third- and fourth-year medical students were comparable in some instances. First, there was no significant difference in the MCQ test scores between the two groups. Also, in the part of the OSCE that focused on the physiology and pathology of cardiovascular physical examination findings, the medical students in both groups obtained similar scores. This could be due to the fact that during their second year of medical training, the medical students had undergone a Harvey simulation course, in which they were taught these topics. Therefore, it is plausible that that the Harvey simulation sessions, together with the integration of their preclinical years with the clinical curriculum, had benefitted the students in applying what they had learnt in preclinical modules to clinical practice.

In the post-examination survey, the third-year medical students were asked about the difficulties faced during the transition from preclinical years to clinical years. Many stated that they lacked confidence in approaching patients and had difficulty remembering the examination steps. Many also mentioned that there was a lack of patients willing to be examined by medical students. However, with the use of Harvey simulation, medical students are given the opportunity to hone their examination technique before approaching real patients. This may help to boost their confidence during clinical rotations. The use of Harvey simulation also alleviates the problem of patient fatigue, as medical students are given the chance to practice on simulators and thus do not require patient contact for every physical examination practice. Other concerns raised by the medical students in the post-examination survey were the short duration and large class size of the simulation sessions. It is advisable to take these points into consideration in future simulation training sessions, so as to optimise the learning of each medical student.

The present study was not without limitations. One obvious limitation is the small number of medical students in each of the groups analysed. This was the case because the study was planned as a pilot study. However, despite the small numbers, significant differences were still observed.

Several studies have shown that deliberate practice leads to expert performance. ${ }^{(1,2)}$ The ability to accurately diagnose a condition hinges on the rarity of the disease and the duration of deliberate practice in appropriate clinical settings. While the use of the Harvey simulator is not meant to replace traditional bedside teaching, it is a potentially useful adjunct in medical education, as it allows hands-on practice in a risk-free environment, boosting students' confidence in their subsequent interaction with real patients. The use of the Harvey simulator is especially useful in helping medical students develop the right examination technique for cardiovascular examination. Lengthening the duration of the Harvey simulation sessions and decreasing the class size may increase the learning value of the lessons.

\section{ACKNOWLEDGEMENTS}

We acknowledge Dr Dujeepa D Samarasekera for his advice and help in the project and in the writing of this paper. We thank Ms Lee Ai Lian and Ms Hazel Phua for their help with the logistics of this study. We also acknowledge Dr Lim Shir Lynn, Dr Low Ting Ting, Dr Kyu Kyu and Dr Charles Ng for their help in conducting the study.

\section{REFERENCES}

1. Duvivier RJ, van Dalen J, Muijtjens AM, et al. The role of deliberate practice in the acquisition of clinical skills. BMC Med Educ 2011; 11:101.

2. Ericsson KA. Deliberate practice and the acquisition and maintenance of 
expert performance in medicine and related domains. Acad Med 2004; 79(10 Suppl):S70-81.

3. Baker GR, Norton PG, Flintoft V, et al. The Canadian Adverse Events Study: the incidence of adverse events among hospital patients in Canada. CMAJ 2004; 170:1678-86.

4. Brennan TA, Leape LL, Laird NM, et al. Incidence of adverse events and negligence in hospitalized patients. Results of the Harvard Medical Practice Study I. N Engl J Med 1991; 324:370-6.

5. Gawande AA, Thomas EJ, Zinner MJ, Brennan TA. The incidence and nature of surgical adverse events in Colorado and Utah in 1992. Surgery 1999; 126:66-75.

6. Vincent C, Neale G, Woloshynowych M. Adverse events in British hospitals: preliminary retrospective record review. BMJ 2001; 322:517-9.

7. Wilson RM, Runciman WB, Gibberd RW, et al. The Quality in Australian Health Care Study. Med J Aust 1995; 163:458-71.

8. Issenberg SB, McGaghie WC, Petrusa ER, Lee Gordon D, Scalese RJ. Features and uses of high-fidelity medical simulations that lead to effective learning: a BEME systematic review. Med Teach 2005; 27:10-28.

9. Butter J, McGaghie WC, Cohen ER, Kaye M, Wayne DB. Simulation-based mastery learning improves cardiac auscultation skills in medical students. J Gen Intern Med 2010; 25:780-5.

10. Ewy GA, Felner JM, Juul D, et al. Test of a cardiology patient simulator with students in fourth-year electives. J Med Educ 1987; 62:738-43.
11. Gordon MS, Ewy GA, DeLeon AC Jr, et al. "Harvey," the cardiology patient simulator: pilot studies on teaching effectiveness. Am J Cardiol 1980; 45:791-6.

12. Horiszny JA. Teaching cardiac auscultation using simulated heart sounds and small-group discussion. Fam Med 2001; 33:39-44.

13. Issenberg SB, Gordon MS, Greber AA. Bedside cardiology skills training for the osteopathic internist using simulation technology. J Am Osteopath Assoc 2003; 103:603-7.

14. Woolliscroft JO, Calhoun JG, Tenhaken JD, Judge RD. Harvey: the impact of a cardiovascular teaching simulator on student skill acquisition. Med Teach 1987; 9:53-7.

15. Lam CS, Cheong PY, Ong BK, Ho KY. Teaching cardiac auscultation without patient contact. Med Educ 2004; 38:1184-5.

16. Teteris E, Fraser K, Wright B, McLaughlin K. Does training learners on simulators benefit real patients? Adv Health Sci Educ Theory Pract 2012; 17:137-44.

17. Karnath B, Thornton W, Frye AW. Teaching and testing physical examination skills without the use of patients. Acad Med 2002; 77:753.

18. Issenberg SB, Pringle S, Harden RM, Khogali S, Gordon MS. Adoption and integration of simulation-based learning technologies into the curriculum of a UK Undergraduate Education Programme. Med Educ 2003; 37 Suppl 1:42-9. 


\section{APPENDIX}

\section{Post-simulation survey}

Instructions

Please indicate by highlighting or circling the appropriate response to the questions below. It is about how YOU perceive the learning experience.

Gender: Male/Female

1. I have gone through the new simulation programme in Phases I and II of the revised undergraduate curriculum.
a. Yes
b. No

If the answer to the above is ' $\mathrm{No}^{\prime}$, please go to question 10 directly. If the answer to the above is 'Yes', please proceed to question 2.

2. How positive were your overall simulation learning experiences in Phases I and II of the curriculum?
a. Very Positive
b. Positive
c. Undecided
d. Negative
e. Very Negative

3. Simulation training sessions have helped me understand Basic Medical Sciences better in Phases I and II.
a. Strongly Disagree
b. Disagree
c. Undecided
d. Agree
e. Strongly Agree

4. Simulation training sessions have helped me to remember the relevant Basic Medical Science concepts/theories better than in modules when these concepts/theories were taught without the linked simulation training sessions.
a. Strongly Disagree
b. Disagree
c. Undecided
d. Agree
e. Strongly Agree

5. Simulation training sessions have helped me to answer scenario-based questions during the exams.
a. Strongly Disagree
b. Disagree
c. Undecided
d. Agree
e. Strongly Agree

6. The simulation training sessions were excellent in training me, preparing me for the examination of patients with similar cardiovascular conditions in future clinical postings.
a. Strongly Disagree
b. Disagree
c. Undecided
d. Agree
e. Strongly Agree

7. The simulation training sessions have made me confident to examine patients with similar cardiovascular conditions when I move into the clinical phase of my learning.
a. Strongly Disagree
b. Disagree
c. Undecided
d. Agree
e. Strongly Agree

8. What do you like most about simulation learning in Phases I and II of the curriculum?

9. What do you like least about simulation learning in Phases I and II of the curriculum?

10. I was confident learning from the examination of patients with cardiovascular conditions when I graduated from medical year 3 to 4 .
a. Strongly Disagree
b. Disagree
c. Undecided
d. Agree
e. Strongly Agree

11. What difficulties did you face when learning from patients (i.e. examining real patients) in the initial transition period from medical year 2 to 3 ? 